\title{
Extension sémantique de lexie chez des apprenants chinois plurilingues
}

\author{
${ }^{1}$ Xian LIU, ${ }^{2}$ Zhihong PU \\ 1,2 Université Sun Yat-sen - Chine \\ Reçu le 17 mai 2019 | Accepté le 30 juin 2019
}

\begin{abstract}
RÉSUMÉ. L'extension sémantique, un genre d'erreur interlinguale dans la production en français (L3) des apprenants chinois dont L2 est l'anglais, présenterait-elle des influences translinguistiques des deux langues : chinois (langue maternelle) et anglais? Telle est la question de cette recherche réalisée par le biais d'une enquête menée auprès de 137 étudiants de français dans quatre universités chinoises, afin de découvrir le mécanisme de mental processing des apprenants plurilingues. Avec les analyses quantitatives (Test-t) et qualitatives des productions écrites et en s'appuyant sur des théories de transfert (Swan, 1997 ; Jiang, 2000 ; Wei, 2009) et la théorie du prototype, on découvre que l'interférence des lemmes plurilingues (chinois-français, anglais-français, chinois-anglais-français) remonte à des extensions sémantiques : Les catégories sémantiques des lemmes n'étant pas les mêmes, les apprenants auraient tendance à construire certains lemmes de L3 (le français dans ce cas) en se balançant entre ceux de L1 et de L2. Ainsi, il faut considérer davantage les influences des langues non-cibles, notamment l'interférence de L2 dans l'enseignement/apprentissage de L3.
\end{abstract}

Mots-clés : Extension sémantique, habileté, influence translinguistique, lemme, source de transfert

\begin{abstract}
Semantic extension, a typical interlingual error in French-language production (L3) of Chinese students (their L2 is English), does it present the cross-linguistic influence of two languages: Chinese (mother tongue) and English? This is the research question, realized by a survey conducted among 137 students of French in four Chinese universities, in order to discover multilingual learners' mental processing mechanism. With quantitative ( $\mathrm{T}$ test) and qualitative analysis of written productions, using the transfer theories (Swan, 1997; Jiang, 2000; Wei, 2009) and prototype theory, the research discovers that the interference of multilingual lemmas (Chinese-French, English-French, Chinese-English-French) accounts for semantic extensions: the learners tend to construct some lemmas of L3 (French in the case) by swaying between lemmas of L1 and L2. Thus, it needs to consider further the influences from non-target languages, particularly the interference of L2 in teaching/learning of L3.
\end{abstract}

Keywords: cross-linguistic influence, lemma, semantic extension, skill, source of transfer

囷 auteur correspondant : puzhihong@hotmail.com

Pour citer cet article (Style APA): Liu \& Pu. (2019). Extension sémantique de lexie chez des apprenants chinois plurilingues. Francisola: Revue Indonésienne de la langue et la littérature françaises, 4(1), 22-29. doi: 10.17509/francisola.v4i1.20338. 


\section{INTRODUCTION}

L'extension sémantique est un genre typique d'erreur interlinguale dans la production des apprenants plurilingues, qui étendent la catégorie sémantique du mot de la langue cible selon celle du mot des autres langues. Certains chercheurs ont observé ce phénomène dans la production lexicale de L2 et ont découvert que l'apprenant tend à utiliser la lexie de L2 en s'appuyant sur la catégorie sémantique de celle de L1 (Ringbom, 1978 ; Biskup, 1992 ; Hasselgren, 1994). Dans le domaine de l'acquisition trilingue, les chercheurs ont commencé à étudier si la catégorie sémantique du mot de L2 peut s'étendre à L3. Par exemple, Ringbom (1987) a trouvé que l'extension sémantique, en tant que transfert de système, ne provient que de la langue maternelle ou de la langue non-maternelle dans laquelle l'apprenant se révèle fort habile. Bouvy (2000) a découvert que l'extension sémantique apparaît davantage chez des apprenants $\mathrm{du}$ niveau linguistique plus élevé.

Néanmoins, les recherches précédentes se limitant à la structure de surface linguistique, ne pénètrent guère dans le mental processing (traitement mental) de la langue. Afin de creuser ce phénomène de transfert, nous explorons du point de vue cognitif et psychologique sa source profonde.

Pour les étudiants chinois de français dans notre recherche, la plupart d'entre eux ont appris l'anglais dès leur $3 e$ année d'études primaires. Ainsi, ce genre d'erreur présenterait-il des influences translinguistiques entre chinois (L1), anglais (L2), français (L3) ? Nous étudions ce problème dans le but de découvrir le mécanisme de mental processing au-dessous des influences translinguistiques du niveau sémantique, en nous appuyant sur des théories de transfert : l'activation des lemmes plurilingues (Wei, 2009), l'hypothèse de l'équivalence (Swan, 1997), le développement lexical de L2 (Jiang, 2000), ainsi que la théorie du prototype. Selon Wei (2009), les concepts à exprimer peuvent activer en parallèle des lemmes de différentes langues, manque d'informations du lemme de la langue cible, l'apprenant transfère celles du lemme correspondant des langues acquises auparavant dans le traitement de la langue. D'après l'hypothèse de l'équivalence, l'apprenant tend à égaliser la sémantique des mots de la langue étrangère et celle des mots de la langue maternelle. Jiang (2000) propose un modèle du développement lexical de L2.

Ce processus connaît trois étapes : de l'association lexical à l'intégration complète par l'intermédiaire de la médiation des lemmes de L1. Selon la théorie du prototype, les signifiés d'un mot polysémique se divisent en signifié prototypique et ceux de marginaux. Les seconds se forment autour du premier. Ces théories constituent le cadre théorique de cette recherche.

\section{MÉTHODE}

En vue de répondre à la question posée, nous avons mené un test avec la traduction du chinois en français auprès de 82 étudiants de première année et de 55 étudiants de troisième année, spécialisés tous en deux orientations: français et anglais (comme cours majeurs) au sein de quatre universités chinoises (Université Sun Yat-sen, Université de Shenzhen, Université du Shanxi et Université Polytechnique de Wuhan). Nous avons sélectionné respectivement 20 phrases et un texte chinois pour les étudiants de première année (niveau élémentaire) et ceux de troisième année (niveau intermédiaire). Tous les enquêtés les ont traduits en français en 30 minutes, sans avoir recouru aux dictionnaires ni à d'autres documents de référence. Après le test, certains enquêtés ont été demandé de refaire cette traduction en anglais pour nous permettre de comparer leurs traductions en anglais et en français.

Nous avons recouru aux analyses quantitatives et qualitatives. Plus spécifiquement, nous utilisons en matière $\mathrm{d}^{\prime}$ analyses quantitatives, $l^{\prime}$ analyse statistique descriptive et le test $\mathrm{T}$ pour échantillons indépendants afin d'explorer le nombre $\mathrm{d}$ 'erreurs et leur répartition en fonction $\mathrm{du}$ niveau de français des étudiants et de celui 
de la langue source (anglais dans notre cas). Dans les analyses qualitatives, nous combinons l'analyse des erreurs avec celle comparative. En comparant de différentes manières d'expression des langues chinoises, anglaise et française, nous analysons les erreurs typiques commises par les enquêtés sous l'interférence translinguistique. Notre objectif vise à étudier, d'une part, la relation entre l'habileté linguistique, les sources de transfert et l'extension sémantique, d'autre part, les causes profondes des erreurs.

\section{RÉSULTATS ET DISCUSSION}

Nos résultats et discussions se divisent

Tableau 1. Répartition des extensions sémantiques

\begin{tabular}{cccccc}
\hline & Niveau & chinois & $\begin{array}{c}\text { Langue source } \\
\text { sino-anglais }\end{array}$ & anglais & Total \\
\hline $\begin{array}{c}\text { Groupe } 1 \\
\mathrm{n}=82\end{array}$ & $\begin{array}{c}\text { Première année } \\
\text { (niveau élémentaire) }\end{array}$ & 42 & 3 & 2 & 47 \\
$\begin{array}{c}\text { Groupe } 2 \\
\mathrm{n}=55\end{array}$ & $\begin{array}{c}\text { Troisième année } \\
\text { (niveau intermédiaire) }\end{array}$ & 7 & 28 & 23 & 58 \\
\hline
\end{tabular}

Dans ces données, les enquêtés de troisième année commettent plus d'erreurs que ceux de première année. Cela a été justifié par le test $\mathrm{T}$ pour échantillons indépendants. La moyenne de chaque groupe pour le nombre d'erreurs est respectivement 0.5732 et $1.0545, \mathrm{P}=0.000$, le degré de signification est plus petit que 0.05 . Les moyennes $(1.0545>0.5732)$ sont significativement différentes. Du point de vue de Ringbom (2001, 2007), l'apprentissage de la langue cible connaît une évolution de la dépendance formelle à celle de sémantique : des apprenants ayant moins d'habileté en langue cible s'appuient davantage sur la similarité morphologique ou phonétique entre langues tandis que ceux qui ont plus en deux parties : l'une comporte les statistiques quantitatives et l'analyse de donnée ; l'autre, l'exploration des sources des erreurs des étudiants.

\subsection{Statistiques quantitatives et répartition des extensions sémantiques}

Nous avons trouvé totalement 105 cas d'extension sémantique dans la production des enquêtés. Le tableau 1 présente leur répartition en fonction du niveau des étudiants et de la langue source.

Tableau 2. Statistiques de moyenne et test d'échantillons indépendants

\begin{tabular}{ccccccc}
\hline $\begin{array}{c}\text { Langue } \\
\text { source }\end{array}$ & Niveau & N & Moyenne & \multicolumn{2}{c}{ Test-t pour égalité des moyennes } \\
$\mathbf{t}$ & & & $\mathbf{d f}$ & Sig. (bilatérale) \\
\hline chinois & Première année & 82 & 0.5122 & \multirow{2}{*}{5.218} & 134.727 & 0.000 \\
& Troisième année & 55 & 0.1273 & & & \\
\multirow{2}{*}{ sino-anglais } & Première année & 82 & 0.0366 & & & 0.000 \\
& Troisième année & 55 & 0.5091 & -6.640 & 64.253 & \\
& Première année & 82 & 0.0244 & & & 0.000 \\
\hline
\end{tabular}


Quand le chinois est la langue source, la moyenne de chaque groupe pour le nombre d'erreurs est respectivement 0.51222 et $0.1273, \mathrm{P}=0.000<0.05$, les moyennes $(0.51222>0.1273)$ sont significativement différentes. C'est-à-dire que les étudiants de première année sont plus influencés par leur langue maternelle. Au contraire, quand la langue source est l'anglais ou le sino-anglais,

chinois

Niveau élémentaire

Niveau intermédiaire les moyennes sont respectivement 0.0244 et $0.4182,0.0366$ et $0.5091, \mathrm{P}=0.000<0.05$, les moyennes $(0.4182>0.0244,0.5091>0.0366)$ sont significativement différentes. Les étudiants de troisième année restent plus influencés par l'anglais. Nous faisons une comparaison entre les sources de transfert en ce qui concerne l'extension sémantique (voir la figure ci-dessous).

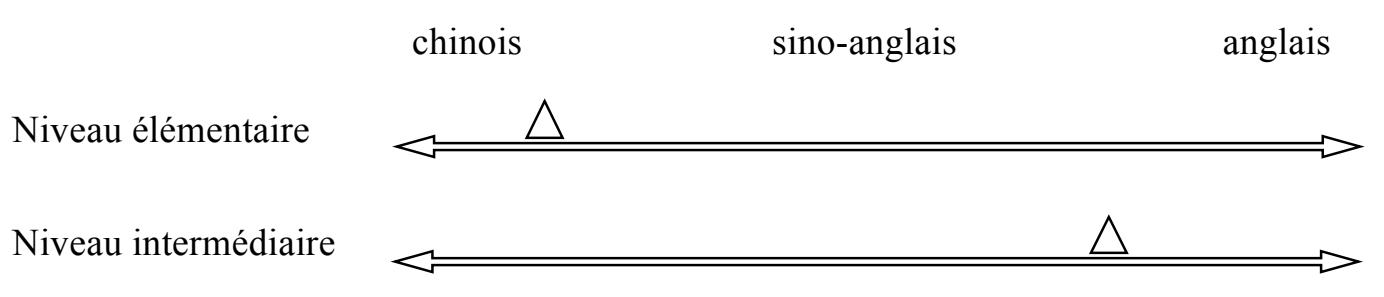

Figure 1. Relation entre l'habileté linguistique et les sources de transfert

Si le chinois et l'anglais se trouvent aux deux pôles, les étudiants de français du niveau élémentaire tendent au chinois, alors que ceux du niveau intermédiaire au milieu de l'anglais et le sino-anglais. Le statut en tant que L2 et le contrôle de L1 se renforcent chez ces derniers.

\subsection{Extension sémantique et l'interférence des lemmes plurilingues}

Notons que l'extension sémantique est le transfert de la catégorie sémantique du mot des langues non-cibles. Du point de vue psychologique, l'interférence des lemmes plurilingue chez des apprenants est à l'origine de ce type d'erreur. D'après Levelt (1989), le lexique mental consiste en informations formelles et éléments abstraits «lemmes», terme introduit par Kempen et Huijbers (1983). Chaque lemme comporte «des spécifications conceptuelles pour son usage (conditions pragmatique et stylistique incluses), et des propriétés (morpho)syntaxiques diverses » (Levelt, 1989, p.233). Autrement dit, le lemme comprend toutes les informations sémantiques, syntaxiques et morphologiques tels personne, temps, cas, aspect, etc. Il demeure au centre du traitement du langage. Chez les plurilingues, tous les lemmes «sont étiquetés comme langue spécifique» (Wei, 2009, p.96). Les concepts à exprimer peuvent activer parallèlement des lemmes de différentes langues. Quand une information sémantique du lemme de la langue cible paraît lacunaire ou incomplète, les informations sémantiques des lemmes concernés des langues acquises précédemment émergent et influent sur l'accès aux mots pertinents, d'où l'extension sémantique dans la production.

Nous analysons ci-après les erreurs typiques commises par nos enquêtés sous l'interférence des lemmes de différentes langues : chinois, anglais ou sino-anglais.

\subsubsection{Langue source de transfert de L1: le chinois}

Les chercheurs parviennent à un consensus quant à l'influence de la langue maternelle sur la sémantique lexicale. La langue maternelle est la première langue que des gens apprennent dès leur enfance. Au cours de l'acquisition de la langue maternelle, ils connaissent le monde et établissent un système conceptuel ainsi qu'un système sémantique y associé. Lorsqu'ils apprennent une nouvelle langue, les concepts et les catégories sémantiques acquis au travers de la langue maternelle peuvent être changés ou augmentés, mais il est impossible de «les remplacer ou éliminer totalement» (Jarvis, 
1998, p.26). En apprenant le français, nos étudiants pensent encore dans leur langue maternelle et sans se débarrasser du système sémantique chinois, comme les deux exemples suivants :

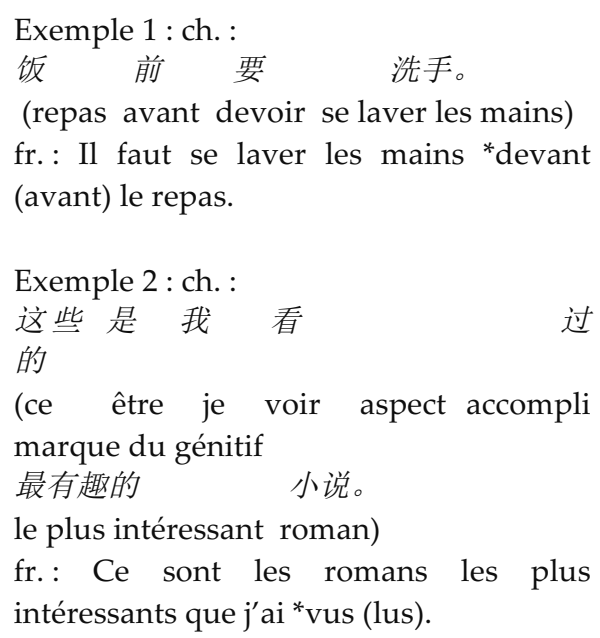

Les erreurs ci-dessus sont dues à l'activation du lemme chinois dans le traitement de la langue française. En chinois, les connotations $\mathrm{d}^{\prime}$ «une antériorité dans le temps ou le classement» et de «du même côté que la face visible ou la façade de quelque chose dans l'espace » s'unissent dans le lemme "前», comme dans les locutions " 午饭前》 (avant le déjeuner) et “大树前” (devant l'arbre), alors qu'en français, le lemme similaire «devant» n'ayant pas la même catégorie sémantique n'exprime que le deuxième sens. C'est le lemme «avant» qui indique le premier. Selon l'hypothèse de l'équivalence (Swan, 1997), les apprenants tendent à égaliser la sémantique des mots étrangers et celle des mots de la langue maternelle. En effet, c'est une stratégie cognitive: favoriser ou simplifier l'acquisition du lexique de L2 en utilisant les connaissances de L1. Dans l'exemple 1, le concept «前» s'active et étend son activation en même temps sur les lemmes correspondants dans le lexique mental. En négligeant la catégorie sémantique du lemme français « devant», l'enquêté y transfère celle du lemme chinois «前», et choisit le mot incorrect «devant» au lieu d' «avant» pour exprimer une antériorité dans le temps.

Dans l'exemple 2, le lemme chinois « 看» exprime le «contact visuel», comme «看
电影》 (voir un film) et 《看书》 (lire un livre). Quoique le lemme français «voir» ait le même signifié, leurs catégories sémantiques sont différentes: on ne dit que «lire un roman» en français. En les confondant, l'apprenant étend la catégorie sémantique du lemme "voir» sous l'influence du lemme « 看», et utilise de manière erronée le verbe «voir» avec le nom «roman» dans la traduction.

\subsubsection{Langue source de transfert de L2 : l'anglais}

Dans le domaine de l'acquisition d'une L3, les chercheurs se concentrent sur l'extension sémantique sous l'influence de L2. Dans notre recherche, les étudiants du niveau élémentaire commettent également ce genre d'erreur, mais le nombre des erreurs est beaucoup moins élevé que celui des étudiants du niveau intermédiaire. Voici trois exemples typiques commis par les enquêtés:

Exemple $3:$ ch. :

她希望得到老板的

(elle espérer acquérir patron marque du génitif admiration)

fr.: Elle espère la *reconnaissance (l'admiration) de son patron.

angl. : She hopes to achieve recognition from her boss.

Exemple 4 : ch. :

每当 我闭上 双眼........

(chaque fois je ferme yeux)

fr.: Chaque*temps (Chaque fois que) je ferme les yeux...

angl. : Each time I close my eyes...

Exemple 5 : ch. :

根据 周五 发布的

一份报告.......

(selon vendredi lancer marque du génitif un rapport)

fr.: Selon le*reportage (rapport) lancé vendredi...

angl. : According to the report launched on Friday...

À notre avis, les apprenants de L3 ont aussi tendance à établir l'équivalence sémantique entre les lexiques des langues non maternelles. C'est-à-dire qu'ils tendent 
également à égaliser la sémantique des mots de L2 et de L3. Comme dans les trois exemples ci-dessus, bien que les mots anglais et français aient des signifiés similaires, leurs catégories sémantiques sont différentes: le lemme anglais «recognition » correspond aux lemmes français «reconnaissance» et «admiration», «time» aux «temps» et «fois», «report» aux «reportage» et «rapport». En fonction des catégories sémantiques des lemmes anglais, les apprenants transfèrent les signifiés dans les lemmes français, d'où l'accès inapproprié aux mots «reconnaissance», «temps» et « reportage ».

Notons que dans ce processus, le concept à exprimer ne réussit pas à activer le lemme correct de L3. C'est un signifié noncible du lemme de L2 qui active le lemme correspondant de L3. Nous devons ainsi poser une question: quel signifié non-cible réalise l'activation du lemme de L3?

Évidemment, seuls les mots polysémiques concernent l'extension sémantique, parce qu'un mot polysémique est une catégorie où divers signifiés associés l'un à l'autre à travers la catégorisation établissent ensemble un réseau, soit la catégorie sémantique du mot. Selon la théorie du prototype, tous les signifiés d'un mot polysémique n'ayant pas le même statut se divisent en signifié prototypique et ceux de marginaux (Wang, 2014, p.472). Le premier est le signifié le plus fondamental, autour duquel se forment les signifiés marginaux. Dans l'accès du mot, il existe la concurrence entre les signifiés parallèlement activés (Fontes, Schwartz, 2015, p.639). Au cours du traitement de la langue, toutes les informations sémantiques $\mathrm{du}$ mot des langues non-cibles s'activent en parallèle, mais leur temps d'activation est différent: l'activation du signifié prototypique est antérieure à celle des signifiés marginaux, et le signifié marginal plus éloigné de celui de prototype s'active plus tard.

En conséquence, parmi les signifiés non-cibles du lemme de L2, les apprenants tendent à activer le lemme de L3 au travers du signifié prototypique qui est activé le plus tôt. Comme dans les exemples 3 et 4, les lemmes français sont activés et choisis en s'appuyant sur les signifiés prototypiques «identification» et «un milieu infini dans lequel se succèdent les événements» des lemmes anglais «recognition» et «time».

$\mathrm{Si}$ le signifié cible est le signifié prototypique du lemme de L2 comme dans l'exemple 5, le signifié le plus proche du signifié prototypique s'active le plus tôt, et étend son activation au lemme correspondant de L3. Ainsi, quand l'apprenant manque le lemme correct "rapport», il tourne vers le lemme anglais «report», dont le signifié non-cible le plus proche du signifié prototypique (article de journal) active le lemme français «reportage». Ensuite, la catégorie sémantique du lemme anglais se transfère dans le lemme français, et le mot «reportage» est choisi pour exprimer le «texte écrit pour rendre compte d'un événement » dans la traduction.

\subsubsection{Langues sources de transfert combiné : le chinois et l'anglais \\ Dans notre recherche, nous} découvrons aussi l'extension sémantique sous l'influence translinguistique combinée, soit une influence commune de L1 et de L2 sur L3. Par exemple :

Exemple $6: \mathrm{ch}$. :

$$
\begin{aligned}
& \text {......科、艺术和 法律 专业 学生。 } \\
& \text { (ingénierie art et droit spécialité } \\
& \text { étudiant) } \\
& \text { fr. : ... les diplômés dans l'ingénierie, l'art } \\
& \text { et la *loi (le droit). } \\
& \text { angl. : ...graduates in engineering, art and } \\
& \text { law. }
\end{aligned}
$$

En chinois et en anglais, les mots «法 律» et «law» indiquent non seulement un ensemble des textes juridiques définissant la légalité ou une certaine règle édictée par l'État, mais aussi la discipline qui étudie l'ensemble des règles juridiques. Cependant, il existe deux mots français correspondants : loi et droit. Dans l'exemple 6, l'enquêté confond les catégories sémantiques de ces mots et choisit la loi au lieu du droit pour exprimer la discipline.

Jiang (2000) propose un modèle du 
développement lexical de L2 (voir la figure 2). Le développement lexical de L2 connaît trois étapes: l'association lexicale, la médiation des lemmes de L1 et l'intégration complète. Dans la première étape, il manque des informations sémantiques, syntaxiques et morphologiques dans les lemmes de L2, les mots de L2 s'utilisent à travers les informations des lemmes de L1. Dans la deuxième étape, l'apprenant transfère des informations des lemmes de L1 dans les lemmes de L2. À la troisième étape, l'apprenant développe complètement les informations des lemmes de L2, en se dégageant de l'interférence des lemmes de L1.

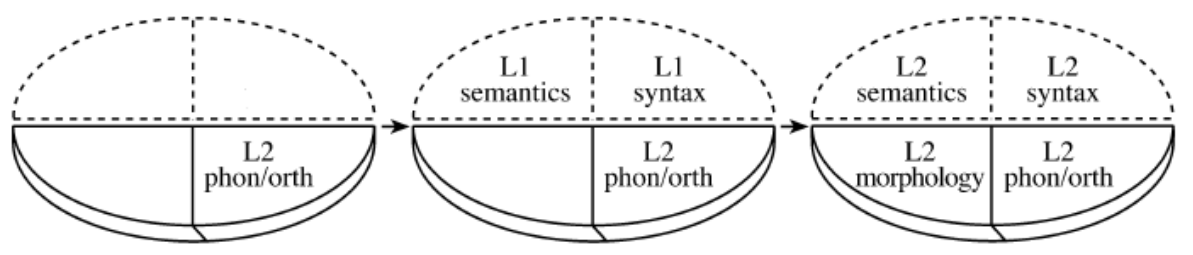

Figure 2. Le développement lexical de L2 (Jiang, 2000)

D'après ce modèle, l'établissement du lemme de L2 «law» se base sur le lemme de L1 《法律》. Étant donné que leurs catégories sémantiques sont similaires, le lemme «law » se développe rapidement. Ainsi la communauté des lemmes de L1 et de L2 renforce-t-elle la cognition de l'apprenant : un signe peut indiquer deux objets: règles juridiques et discipline. Au cours de l'apprentissage de L3, l'apprenant transfère les informations sémantiques communes des lemmes de L1 et de L2 dans le lemme de L3. Son développement lexical de L3 connaît la médiation des lemmes de L1 et de L2. En conséquence, dans le traitement du langage, le concept «discipline qui étudie les règles juridiques» active le lemme «loi » qui réalise l'accès du mot inapproprié dans la traduction.

\section{CONCLUSION}

En nous appuyant sur les théories de transfert : l'activation des lemmes plurilingues (Wei, 2009), l'hypothèse de l'équivalence (Swan, 1997), le développement lexical de L2 (Jiang, 2000), ainsi que la théorie du prototype, nous avons analysé l'extension sémantique de lexie dans la production en français (L3) des apprenants chinois dont L2 est l'anglais.

Avec l'analyse quantitative des productions écrites des enquêtés, nous remarquons que l'extension sémantique arrive davantage chez des étudiants de français du niveau intermédiaire que ceux $d u$ niveau élémentaire, du fait que le transfert basé sur la similarité sémantique demande plus d'habileté en langue cible. Du point de vue des sources de transfert, les étudiants de première année restent plus influencés par leur langue maternelle, le chinois, alors que ceux de troisième année par celles de l'anglais. Au fur et à mesure du développement de l'habileté en L3, l'inhibition de L1 et le statut en tant que L2 se renforcent.

À partir des exemples produits par les enquêtés, nous découvrons que l'interférence des lemmes plurilingues remonte à des extensions sémantiques. Chaque langue a son système sémantique, il en résulte que les catégories sémantiques des lemmes ne sont pas les mêmes. Dans le traitement de la langue, quand les informations sémantiques d'un lemme de L3 sont faibles, l'apprenant recourt aux autres langues apprises auparavant, et a une tendance à établir l'équivalence sémantique entre les mots de différentes langues, il transfère ainsi les catégories sémantiques des lemmes de L1 ou de L2 dans celui de L3. Par ailleurs, quant à l'activation d'un lemme de L3, le signifié prototypique ou celui qui est le plus proche $\mathrm{du}$ signifié prototypique du lemme des langues non-cibles, s'active le plus tôt, et étend son activation au lemme correspondant de L3.

De plus, nous avons trouvé l'extension sémantique sous l'influence translinguistique combinée : les lemmes de L1 et de L2 ayant les mêmes catégories sémantiques collaborent pour une influence sur le lemme de L3. Le système sémantique des étudiants 
se base sur leur langue maternelle, les lemmes de L2 s'établissent par l'intermédiaire des lemmes de L1. Quand les catégories sémantiques des lemmes de L1 et de L2 sont les mêmes, le lien entre les concepts et le signe linguistique se renforce dans la cognition des étudiants, qui le transfèrent dans le développement lexical de L3.

En bref, notre analyse a approfondi notre connaissance sur l'interférence des L1 et L2 dans l'apprentissage de L3. Dans la production langagière des apprenants plurilingues, le lemme joue un rôle important vu l'extension sémantique. Nous devons considérer davantage ce rôle dans l'enseignement / apprentissage de L3.

\section{REMERCIEMENTS}

$\mathrm{Pu}$ Zhihong, auteur de correspondance (tongxunzuozhe) et Liu Xian voudraient bien exprimer leur profonde gratitude à Mesdames Yang Tingfang, Bai Yanyuan, Meng Xiaoqi, Liu Shuqin et Monsieur Xiong Wei, qui ont aidé à la réalisation du test en classe, et remercient également le Bureau de l'Education de Guangdong de nous soutenir dans le projet de Guangdong sheng jiao yu ting jiao yu jiao xue gai ge xiang mu «Stratégies métacognitives et apprentissage en autonomie », yue gao jiao han 2018 n¹80.

\section{RÉFÉRENCES}

Biskup, D. (1992). L1 influence on learners' renderings of English collocations : A Polish/German empirical study. In Pierre, J., Arnaud, L. et Béjoint, H. (eds.), Vocabulary and Applied Linguistics, 85-93. London : Macmillan.

Bouvy, C. (2000). Towards the construction of a theory of cross-linguistic transfer. In Cenoz, J. et Jessner. U. (eds.), English in Europe: The Acquisition of a Third Language, 143-156. Clevedon : Multilingual Matters.

Fontes, A. B. A. D. L. et Schwartz, A. I. (2015). Bilingual access of homonym meanings : Individual differences in bilingual access of homonym meaning. Bilingualism : Language and Cognition, 18(4), 639-656.
Hasselgren, A. (1994). Lexical teddy bears and advanced learners : A study into the ways of Norwegian students cope with English vocabulary. International Journal of Applied Linguistics, 4(2), 237258.

Jarvis, S. (1998). Conceptual transfer in interlingual lexicon. Bloomington : Indiana University Linguistics Club Publications.

Jiang, N. (2000). Lexical representation and development in a second language. Applied Linguistics, 21(1), 47-77.

Kempen, G. et Huijbers, P. (1983). The lexicalization process in sentence production and naming : Indirect election of word. Cognition, 14(2), 185209.

Levelt, W. (1989). Speaking: From Intention to Articulation. Cambridge, MA : The MIT Press.

Ringbom, H. (1978). The influence of the mother tongue on the translation of lexical items. Interlanguage Studies Bulletin, Utrecht, 3 (1), 80-101.

Ringbom, H. (1987). The Role of the First Language in Foreign Language Learning. Clevedon. UK: Multilingual Matters.

Ringbom, H. (2001). Lexical transfer in L3 production. In Cenoz, J., Hufeisen, B. et Jessner, U. (eds.), Cross-linguistic influence in third language acquisition: psycholinguistic perspectives, 59-68. Clevedon : Multilingual Matters.

Ringbom, H. (2007). Cross-linguistic similarity in foreign language learning. Clevedon : Multilingual Matters.

Swan, M. (1997). The influence of the mother tongue on second language vocabulary acquisition and use. In Schmitt, N. et McCarthy, M. (eds.), Vocabulary, description, acquisition and pedagogy, 156-180. Cambridge : Cambridge University Press.

Wang, Y. (2014). Researches on Philosophy of Language: Meditation on China's PostPhilosophy of Language. Beijing, China : Peking University Press.

Wei, L. (2009). Intrasentential codeswitching : bilingual lemmas in contact. Concentric : Studies in Linguistics, 35(2), 307-344. 